\title{
Anacardium occidentale $L$. extract in cosmetic formulations: benefits for oily skin
}

\author{
Extrato de Anacardium occidentale L. em formulações cosméticas: benefícios para a pele \\ oleosa
}

\author{
Daiane G. Mercurio, Tais A. L. Wagemaker, Patrícia M.B.G. Maia Campos \\ Faculty of Pharmaceutical Sciences, University of São Paulo, Avenida do Café, s/n, Ribeirão Preto, SP, Brazil, 14040-903 \\ E-mail: pmcampos@usp.br
}

\begin{abstract}
Cashew (Annacardium occidentale L, family: Anacardiaceae.) is a native plant from tropical America, and is widely cultivated in Asia and Africa. It has great socioeconomic importance for Brazil and for many other countries. Cashew apple (Anacardium occidentale L.,) extract (CAE) is rich in tannins, flavonoids, amino acids, anacardic acids and vitamin $\mathrm{C}$, which can provide desirable benefits to oily skin. The aim of this study was to assess the antioxidant activity of CAE and the improvement of oily skin after application of a sunscreen containing this extract. The antioxidant activity of CAE was evaluated by chemioluminescence assay. A sunscreen was developed and added (FC), or not (F), with 3.5\% of CAE. Transepidermal water loss, stratum corneum water content, sebum content and skin microrelief were evaluated before and after two and four hours of a single application of the F or FC formulations. FC effects on skin were also evaluated after a 28 day-period of twice daily applications on the faces of 12 volunteers. Skin imaging techniques were used to evaluate skin pores and comedones, and sensory analysis was performed. CAE showed antioxidant activity and the FC formulation controlled the sebum secretion after two and four hours. FC reduced the number of skin pores (24\%) and did not increase the number of comedones. These results indicate that $\mathrm{CAE}$ is an innovative active ingredient from Brazilian biodiversity with antioxidant activity and efficacy to improve oily skin conditions.
\end{abstract}

Keywords: Cashew extract, sunscreen, clinical efficacy, antioxidant activity, cosmetics.

\section{Resumo}

O caju (Annacardium occidentale L, família: Anacardiaceae.) é uma planta nativa da América tropical, e é amplamente cultivada na Ásia e África. Tem grande importância socioeconômica para o Brasil e para diferentes países. O extrato de caju (Anacardium occidentale L.) (CAE) é rico em taninos, flavonoides, aminoácidos, ácidos anacárdicos e vitamina $\mathrm{C}$ que podem proporcionar benefícios para a pele oleosa. $\mathrm{O}$ objectivo deste estudo foi avaliar a actividade antioxidante do CAE e a melhoradas condições da pele oleosa após aplicação de protetorsolar contendo este extrato. A actividade antioxidante de CAE foi avaliada por ensaio de quimioluminescência. Um protetor solar foi desenvolvido e adicionado (FC) ou não (F) com 3,5\% de CAE. A perda transepidérmicade água,o teor de água do estrato córneo, o conteúdo de sebo e o microrrelevo da pele foram avaliados antes e após 2 e 4 horas de uma única aplicação de F e FC; e após um período de 28 dias de aplicações duas vezes ao dia na face de 12 voluntárias. Técnicas de imagem cutânea foram utilizadas para avaliar poros e comedões. A análise sensorial também foi realizada. O CAE mostrou actividade antioxidante. FC controlou a secreção de sebo após 2 e 4 horas, reduziu o número de poros da pele (24\%) e não aumentou o número de comedões. O CAE é um ingrediente ativo inovador da biodiversidade brasileira com actividade antioxidante e eficácia para melhorar as condições da pele oleosa.

Palavras-chave: Extrato de caju, protetor solar, eficácia clínica, actividade antioxidante, cosméticos. 


\section{Introduction}

An oily skin type is characterized by excess of sebum production and is related to clinical signals such as comedones, papules and enlarged skin pores. The over-production of sebum causes a hydrolipidic mantle imbalance which can have a negative impact on quality of life.

As for all skin types, oily skin also needs photoprotection. However, the high content of oil in sunscreen formulations with high Sun Protection Factor (SPF) presents a challenge.

Cashew (Annacardium occidentale L, family: Anacardiaceae.) is a native plant from tropical America, and is widely cultivated in Asia and Africa. It has great socioeconomic importance for Brazil and as well as for other countries.. Cashew cultivation occupies an estimated 750,000 hectares with a cashew nut production of 280,000 tons/year ${ }^{1}$. However, the potential utility of Cashew apple has not been well explored considering the abundance of available raw material.

The cashew consists of distinct parts, the "cashew apple", a fleshy pseudofruit with a brilliant yellow or red skin, and a nut of grey/brown colour which hangs from the end of the apple and from which the kernel is extracted. The cashew nut and the liquid extracted from its bark are widely exported. The cashew apple pseudofruit is considered a by-product, and is used in food industry for production of juices and candies. As with other agro-industrial by-products, cashew apple has the potential to be used in cosmetic products, providing economic advantage ${ }^{2}$.

The cashew apple may exhibit an acrid taste due to the presence of some phenolic compounds at the mature stage, i.e. when nuts are collected, which can limit their use and export ${ }^{3,4}$.

The extract obtained from Cashew pseudofruit is rich in anacardic acids, resorcinolic acid, carotenoids ( $\alpha$-carotene, $\beta$-carotene and $\beta$-cryptoxanthin), flavonoids, amino acids, minerals and vitamins, including vitamin C. It also contains high concentration of tannins. Tannins are astringent compounds, which have been shown to have an effect on the regulatory activity of the sebaceous secretions and constriction of skin pores $^{5,6}$. The combination of these compounds can provide unique benefits to skincare formulations ${ }^{2,7,8,9}$.

Stimulation of DNA repair or reversal of DNA damage and suppression of mutagenic effects of hydrogen peroxide $\left(\mathrm{H}_{2} \mathrm{O}_{2}\right)$ of Cashew apple juice have also been reported ${ }^{7,10}$.

The anacardic acids are found in small amounts in the cashew pseudofruit. Several biological activities have

\section{Introdução}

O tipo de pele oleosa é caracterizado por excesso de produção de sebo e, está relacionado a sinais clínicos como comedões, pápulas e poros da pele alargados. A produção excessiva de sebo causa um desequilíbrio do manto hidrolipídico que tem impacto negativo na qualidade de vida. Como os outros tipos de pele, a pele oleosa também precisa de fotoproteção. No entanto, o alto teor de óleo em formulações de protetor solar com alto Fator de Proteção Solar (FPS) é um desafio.

O caju (Annacardium occidentale L, família: Anacardiaceae.) é uma planta nativa da América tropical, e é amplamente cultivada na Ásia e África. Tem grande importância socioeconômica para o Brasil e para diferentes países. O cultivo do caju ocupa cerca de $750 \mathrm{mil}$ hectares com uma produção de castanha de caju de 280 mil toneladas/ano ${ }^{(1)}$. No entanto, o potencial de caju não é bem explorado considerando a abundância de matéria-prima disponível.

O caju é composto por partes distintas, a polpa, ou pseudofruto, uma parte suculenta com uma pele brilhante amarela ou vermelha e uma castanha de cor cinza/marrom que é ligada à ponta da polpa e de onde a semente é extraída. A castanha de caju e o líquido extraído de sua casca são amplamente exportados, e o pseudofruto é considerada um subproduto, usado na indústria de alimentos para a produção de sucos e doces. Como os outros subprodutos agroindustriais, a maçã de caju tem potencial para ser utilizada em produtos cosméticos, fornecendo vantagem económica ${ }^{(2)}$.

A maçã de caju pode apresentar um sabor acre devido à presença de alguns compostos fenólicos no estádio de maturação; quando as castanhas são colhidas, o que pode limitar a sua utilização e exportação ${ }^{(3,4)}$.

O extrato obtido a partir do pseudofruto do cajueiro é rico em ácidos anacárdicos, ácido resorcinólico, carotenoides ( $\alpha$-caroteno, $\beta$-caroteno e $\beta$-criptoxantina), flavonoides, aminoácidos, minerais, vitamina $\mathrm{C}$ e outras vitaminas. Também contém alta concentração de taninos. Os taninos são compostos adstringentes, que estão relacionados com a atividade reguladora da secreção sebácea e constrição dos poros cutâneos ${ }^{(5,6)}$. A combinação destes compostos pode proporcionar benefícios únicos para formulações de cuidados da pele $\mathrm{e}^{(2,7,8,9)}$.

A estimulação da reparação do DNA ou reversão da lesão do DNA e a supressão da mutagenicidade dos efeitos do peróxido de hidrogénio $\left(\mathrm{H}_{2} \mathrm{O}_{2}\right)$ do suco de caju foram relatados ${ }^{(7,10)}$.

Os ácidos anacárdicos são encontrados em pequenas quantidades no pseudofruto do caju. Várias actividades biológicas foram atribuídas a estes produtos químicos 
been attributed to these chemicals for the inhibition of activity of important enzymes such as tyrosinase, lipoxygenase ${ }^{11}$. Moreover, the anacardic acids present antimicrobial activity against Gram-positive bacteria such as Staphylococcus aureus and Propionibacterium ac$n e s^{12}$. These activities can be very useful for prevention of acne when the extract is applied in photoprotective formulations for oily skin.

The cashew apple extract (CAE) has constituents that provide effects such as astringency and antimicrobial activity which can provide oil control and acne prevention. This way, it can be considered as a potential active ingredient to be used in sunscreen formulations for oily skin, with an aim to enhance their acceptability.

Thus, the purpose of this study was to assess the antioxidant activity of cashew extract and to evaluate the clinical efficacy of a sunscreen formulation containing this extract to improve oily skin conditions.

\section{Material and Methods}

\section{Anacardium occidentale L. extract}

The extract of Cashew apple (Anacardium occidentale L.) was provided by Mapric ${ }^{\circledR}$ Pharmaceutical and Cosmetic products. The extract was obtained using $20 \%$ of cashew pulp, which was macerated in extraction liquid between 8 and 12 hours and subsequently passed through a percolation process and subsequent filtration. A preservative was added at the end of the process. The extraction liquid comprises of mixture of water (25\%), propylene glycol (70\%) and ethanol (5\%).

\section{Antioxidant activity assay}

In vitro antioxidant activity was measured by the chemiluminescence assay ${ }^{13,14}$. Sample solutions (10 ul each) containing $\mathrm{CAE}$ or positive control solution at a concentration of $0.1 \% \mathrm{w} / \mathrm{w}$ were mixed with $0.1 \mathrm{M}$ phosphate buffer ( $\mathrm{pH} 7.4$ ), followed by the addition of $2 \mathrm{mg} /$ $\mathrm{mL}$ luminol to yield a final concentration of $1.13 \times 10^{-4}$ M. $\mathrm{H}_{2} \mathrm{O}_{2}$ was then added to a final $5 \times 10^{-5} \mathrm{M}$ concentration. The positive control solution yields $100 \%$ of freeradical production and contains no active antioxidant substances. The reaction was initiated by the addition of horseadish peroxidase (HRP) at a final concentration of $0.2 \mathrm{UI} / \mathrm{mL}$ with a final solution volume of $1.0 \mathrm{~mL}$. Chemiluminescence experiments were done in an $\mathrm{Au}-$ tolumat LB953 apparatus (EG \& G Berthold, U.S.A.). The integrated area of chemiluminescence, i.e. the area under the time-course curve of chemiluminescence (AUC), is reported as mean $\pm \mathrm{SEM}$ of three determina- como inibição de enzimas importantes, tais como tirosinase, actividade de lipoxigenase ${ }^{(11)}$. Além disso, os ácidos anacárdicos apresentam actividade antimicrobiana contra bactérias Gram-positivas tais como Staphylococcus aureus e Propionibacterium acnes ${ }^{(12)}$. Estas actividades podem ser muito úteis para a prevenção da acne, quando o extrato é aplicado em formulações fotoprotectoras para pele oleosa.

$\mathrm{O}$ extrato de caju (CAE) tem constituintes que proporcionam efeitos como adstringencia e atividade antimicrobiana, os quais podem proporcionar controloda oleosidade e prevenção da acne. Portanto, este pode ser adicionado a protetores solares indicados para a pele oleosa com o objetivo de aumentar a sua aceitabilidade Assim, o objetivo deste estudo foi avaliar a actividade antioxidante do extrato de caju e avaliar a eficácia clínica de uma formulação de filtro solar contendo este extrato para melhorar as condições da pele oleosa.

\section{Material e métodos}

\section{Anacardium occidentale L. extrato}

O extrato de caju (Anacardium occidentale L.) foi fornecido por Mapric ${ }^{\circledR}$ Farmacêuticos e Cosméticos. O extrato foi obtido utilizando $20 \%$ de polpa de caju, que foi macerada num líquido de extração entre 8 e 12 horas e subsequentemente submetida a um processo de percolação e subsequente filtração. No final, o conservante foi adicionado. O líquido de extração compreende uma mistura de água (25\%), propileno glicol $(70 \%)$ e etanol $(5 \%)$.

\section{Ensaio de actividade antioxidante}

A actividade antioxidante in vitro foi medida pelo ensaio de quimioluminescência ${ }^{(13,14)}$. Uma aliquota de $10 \mu \mathrm{L}$ das amostras de soluções (concentração final de $0,1 \%, \mathrm{p} / \mathrm{p}$ ) contendo CAE ou solução de controlo positivo (sem qualquer substância activa, que produz 100\% de produção de radicais livres ) com tampão fosfato 0,1 $\mathrm{M}(\mathrm{pH} 7,4)$ foram adicionadas a solução de luminol a $2 \mathrm{mg} / \mathrm{mL}$ para se obter uma concentração final de 1,13 X $10^{-4} \mathrm{M}$. Adicionou-se $\mathrm{H}_{2} \mathrm{O}_{2}$ a uma concentração final de $5 \times 10^{-5} \mathrm{M}$. A reacção foi iniciada pela adição de horseadish peroxidase (HRP) a uma concentração final de $0,2 \mathrm{UI} / \mathrm{mL}$ e completando o volume final da solução para 1,0 mL. Os experimentos de quimioluminescência foram realizados num aparelho Autolumat LB953 (EG \& G Berthold, E.U.A.). A área integrada de quimioluminescência, ou seja, a área sob a curva tempo-curso de quimioluminescência (AUC), é relatada como média \pm SEM de 3 determinações e representa o total de espé- 
tions and represents the total of radical oxygen species (ROS) produced in the sample in 15 minutes at $30^{\circ} \mathrm{C}$.

\section{Sunscreen formulation}

The experimental formulation contained Sodium Polyacrylate, Dimethicone, Cyclopentasiloxane, Trideceth-6, PEG/PPG-18/18 Dimethicone (Dow Corning Corporation, Salzburg, USA), Acrylates/C10-30 Alkyl Acrylate Crosspolymer (The Lubrizol Corporation, Wickliffe, USA) containing bis-ethylhexyloxyphenol methoxyphenyl triazine (BASF SE, Ludwigshafen, Germany), Diethylamino hydroxybenzoyl hexyl benzoate (BASF SE, Ludwigshafen, Germany), Ethylhexyl Triazone (BASF SE, Ludwigshafen, Germany), Ethylhexyl Methoxycinnamate (BASF SE, Ludwigshafen, Germany) and Titanium Dioxide (Juk, Galena, Campinas, Brazil) as UV filters. The formulation was supplemented (FC) or not (F), with $3.5 \%$ of $\mathrm{CAE}^{15}$. This formulation was prepared using a mechanical stirrer $\left(\right.$ Heidolph $^{\circledR}$, RZR 2021) for 20 minutes at $600 \mathrm{rpm}$.

\section{Preliminary stability tests}

The formulations under study - F and FC- were subjected to preliminary stability tests by centrifugation (Excelsa Baby II, 206-R, Fanem), pH determination (DM 20 , Digimed), and visual evaluation of the organoleptic characteristics for 28 days. Organoleptic characteristics, $\mathrm{pH}$ and centrifugation did not show any significant differences after 28 days under heat stress at $37^{\circ} \mathrm{C}$ and $45^{\circ} \mathrm{C}$, with controlled humidity and photoperiod.

\section{Study protocol}

Twelve female volunteers aged between 18-30 years (mean age $25.4 \pm 3.2$ ) having skin Fitzpatrick types II and III were enrolled. Subjects were randomly assigned to receive the formulation containing active ingredients. All subjects signed informed consent forms approved by the University of São Paulo Ethical Committee (CEP/FCFRP 234/2011), and the study protocol conformed to the principles set forth by the Declaration of Helsinki. The inclusion criteria included having oily skin on the face, with quantity of sebum on the forehead $\geq 150 \mu \mathrm{g} / \mathrm{cm}^{2}$ as evaluated by Sebumeter ${ }^{\circledR}$ (Courage \& Khazaka, Cologne, Germany).

Prior to all measurements, subjects were instructed to wash their faces two hours before the measurements and to remain on the laboratory premises for at least 15 minutes for full skin adaptation to room temperature $\left(22 \pm 2^{\circ} \mathrm{C}\right)$ and humidity $(45-60 \%)^{16}$. cies radicais de oxigénio (ROS) produzidas no meio em $15 \min$ a $30^{\circ} \mathrm{C}$.

\section{Formulação fotoprotetora}

A formulação experimental continha Poliacrilato, Dimeticona, Ciclopentaciloxano, Trideceth-6, PEG/PPG18/18 Dimeticona (Dow Corning Corporation, Salzburg, EUA), Copolímero de acrilatos/C10-30 alquil acrilato (The Lubrizol Corporation, Wickliffe, EUA) containing Bis-etil-hexiloxifenol metoxifenil triazina (BASF SE, Ludwigshafen, Alemanha), Dietilamino hidroxibenzoil hexil benzoato (BASF SE, Ludwigshafen, Alemanha), Etil-hexil triazona (BASF SE, Ludwigshafen, Alemanha), Etil-hexil metoxicinamato (BASF SE, Ludwigshafen, Alemanha) e Dióxido de titânio (Juk, Galena, Campinas, Brasil) como filtros UV. A formulação foi adicionada (FC) ou não (F), com 3,5\% de $\mathrm{CAE}^{(15)}$. Esta formulação foi preparada utilizando o agitador mecânico Heidolph ${ }^{\circledR}$, RZR 2021, durante 20 minutos, a $600 \mathrm{rpm}$.

\section{Testes preliminares de estabilidade}

As formulações em estudo - F e FC - foram submetidas a testes preliminares de estabilidade por centrifugação (Excelsa Baby II, 206-R, Fanem), determinação do pH (DM 20, Digimed) e avaliação visual das características organoléticas por 28 dias. As características organolépticas, $\mathrm{pH}$ e centrifugação não mostraram diferenças significativas após 28 dias sob estresse térmico a 37 e $45^{\circ}$ $\mathrm{C}$, com humidade controlada e fotoperíodo.

\section{Protocolo de estudo}

Doze voluntárias do sexo feminino com idade entre 1830 anos (média de idade 25,4 $\pm 3,2$ ) com pele Fitzpatrick tipos II e III foram recrutadas. Elas foram distribuídas aleatoriamente para receber a formulação contendo os ingredientes activos. Todas as voluntárias assinaram o termo de consentimento livre e esclarecido aprovado pelo Comitê de Ética da Universidade de São Paulo (CEP / FCFRP 234/2011) eo protocolo do estudo seguiu os princípios estabelecidos na Declaração de Helsínquia. Os critérios de inclusão incluíram a presença de pele oleosa na face, com quantidade de sebo na testa $\geq 150 \mu \mathrm{g} / \mathrm{cm}^{2}$ avaliada pelo Sebumeter ${ }^{\circledR}$ (Courage \& Khazaka, Colônia, Alemanha).

Antes de todas as medições, as voluntárias foram instruídas a lavar o rosto duas horas antes das medições e permaneceram no local durante pelo menos 15 minutos para a adaptação total da pele à temperatura ambiente $\left(22 \pm 2{ }^{\circ} \mathrm{C}\right)$ e humidade $(45-60 \%)^{(16)}$. 


\section{Effects after a single application}

The objective evaluation was made in regard tosebum content (Sebumeter ${ }^{\circledR}$ Courage \& Khazaka, Cologne, Germany) and sebaceous gland activity (Sebufix ${ }^{\circledR} \mathrm{F} 16$, Courage \& Khazaka, Cologne, Germany). Measurements were carried out before application of any formulation and two and hour hours after application of $12 \mu \mathrm{l}$ of the formulations $\mathrm{F}$ and $\mathrm{FC}$ on a $2 \mathrm{~cm}^{2}$ region of the forehead, and one on the region used as control (no treatment). The measurements of transepidermal water loss (TEWL) (Tewameter ${ }^{\mathbb{B}}$ TM 300, Courage \& Khazaka, Cologne, Germany), stratum corneum water content (Corneometer ${ }^{\circledR}$ CM 825, Courage \& Khazaka, Cologne, Germany), and skin microrelief (Visioscan ${ }^{\circledR}$ VC 98, Courage \& Khazaka, Cologne, Germany) were performed before and after (two and four hours following) application of $50 \mu \mathrm{l}$ of the formulations $\mathrm{F}$ and FC on a $3 \mathrm{~cm}^{2}$ region on the malar area ${ }^{16}$.

\section{Long term effects}

The long term effects were evaluated by objective measurements in terms of transepidermal water loss (TEWL) (Tewameter ${ }^{\circledR}$ TM 300), stratum corneum water content (Corneometer ${ }^{\circledR} \mathrm{CM} 825$ ), sebum content $\left(\right.$ Sebumeter $\left.^{\mathbb{R}}\right)$, sebaceous gland activity (Sebufix $\left.{ }^{\mathbb{B}} \mathrm{F} 16\right)$ and skin microrelief (Visioscan ${ }^{\circledR}$ VC 98) ${ }^{16}$.

After the baseline measurements, approximately $0.1 \mathrm{~g} /$ $\mathrm{cm}^{2}$ of formulation FC was applied to the face of each subject, twice daily, during 28 days. The measurements were repeated 24 hours after the last application.

\section{Skin comedogenicity tests}

Skin imaging techniques (Visioface ${ }^{\circledR}$, Visioscan ${ }^{\circledR}$ VC 98 and Vivascope ${ }^{\circledR} 1500$ ) were used to evaluate comedones and skin pores on the cheeks and $\operatorname{chin}^{16}$ before, and after 28-day-period of twice daily applications of the formulation containing CAE.

\section{Sensory evaluation}

For the sensory analysis, $50 \mu \mathrm{l}$ of each formulation were applied on the volar forearm of the 12 volunteers. They were instructed to spread the formulation by hand? on a $5 \mathrm{~cm}^{2}$ square region with ten circular movements. They were then asked to answer a questionnaire about their perception concerning sensation to touch, sensation to touch after 5 minutes and purchase intention ${ }^{17}$.

\section{Statistical analysis}

In order to evaluate the normal distribution of the experimental data from the formulations' effects on hu-

\section{Efeitos após uma única aplicação}

A avaliação objetiva foi feita em termos de conteúdo de sebo (Sebumeter ${ }^{\circledR}$ Courage \& Khazaka, Colônia, Alemanha) e atividade da glândula sebácea (Sebufix ${ }^{\circledR}$ F16, Courage \& Khazaka, Colônia, Alemanha). As medições foram realizadas antes e após 2 e 4 horas de aplicação, $12 \mu \mathrm{l}$ das formulações $\mathrm{F}$ e FC numa região de $2 \mathrm{~cm}^{2}$ da testa e uma na região utilizada como controlo (sem tratamento). As medições de perda transepidérmica de água (PTEA) (Tewameter ${ }^{\circledR}$ TM 300, Courage \& Khazaka, Colónia, Alemanha), conteúdo de água do estrato córneo (Corneometer ${ }^{\circledR} \mathrm{CM} 825$, Courage \& Khazaka, Colónia, Alemanha) e microrrelevo da pele (Visioscan ${ }^{\circledR}$ VC 98, Courage \& Khazaka, Colónia, Alemanha) antes e após 2 e 4 horas de aplicação de $50 \mu \mathrm{l}$ das formulações F e FC numa região de $3 \mathrm{~cm}^{2}$ na região malar ${ }^{(16)}$.

\section{Efeitos de longo prazo}

Os efeitos a longo prazo foram avaliados por medidas objetivas em termos de perda transepidérmica de água (PTEA) (Tewameter ${ }^{\circledR}$ TM 300), conteúdo de água no estrato córneo (Corneometer $\left.{ }^{\circledR} \mathrm{CM} 825\right)$, conteúdo de sebo (Sebumeter ${ }^{\circledR}$ ), actividade da glândula sebácea (Sebufix ${ }^{\circledR} \mathrm{F} 16$ ) e microrrelevo da pele (Visioscan ${ }^{\circledR} \mathrm{VC}$ $98)^{(16)}$.

Após as medidas basais, aproximadamente $0,1 \mathrm{~g} / \mathrm{cm}^{2} \mathrm{de}$ formulação FC foi aplicado à face de cada indivíduo, duas vezes ao dia, durante 28 dias. As medições foram repetidas 24 horas após a última aplicação.

\section{Testes de comedogenicidade cutânea}

Foram utilizadas técnicas de análise de imagem da pele (Visioface ${ }^{\circledR}$, Visioscan ${ }^{\circledR}$ VC 98 e Vivascope ${ }^{\circledR} 1500$ ) para avaliar comedões e poros da pele nas bochechas e quei$\mathrm{xo}^{(16)}$ antes e após 28 dias de aplicação duas vezes ao dia da formulação contendo CAE.

\section{Avaliação sensorial}

Para a análise sensorial, $50 \mu 1$ de cada formulação foram aplicados na parte interna do antebraço das 12 voluntárias. Elas foram instruídas a espalhar a formulação numa região quadrada de $5 \mathrm{~cm}^{2} \mathrm{com}$ dez movimentos circulares. Elas foram então convidados a responder a um questionário sobre sua perceção quanto a sensação ao toque, sensação ao toque após 5 minutos e intenção de compra ${ }^{(17)}$.

\section{Análise estatística}

Para avaliar a distribuição normal dos dados experimentais a partir dos efeitos das formulações sobre a pele 
man skin the normality test Kolmogorov-Smirnov was used using statistical software Origin 9.0. For the effects after a single application and subjective analysis, data was analyzed using T- Student test for normal distribution and Mann Whitney test for nonparametric distribution. For the long term effects, ANOVA parametric analysis with Tukey's post-test were used, and for nonparametric distribution, the Kruskal-Wallis with Dunns posttest were used. The above tests were performed using GraphPad Prism 5.0. Differences were accepted as statistically significant at $\mathrm{p}<0.05$.

\section{Results}

\section{Antioxidant activity}

CAE inhibited the Chemiluminescence catalized by HRP in a dose dependent manner and demonstrated radical scavenging activity (Figure 1), with a concentration value of $8.63 \mathrm{mg} / \mathrm{mL}$ for $50 \%$ inhibition of free radicals $\left(\mathrm{IC}_{50}\right)$.

\section{Clinical efficacy}

No significant immediate effects were obtained in stratum corneum water content, transepidermal water loss and microrelief parameters. The application of formulation $\mathrm{F}$ (without CAE) increased the sebum level twoand four hours after application (Figure 2). However, in spite of having the same UV filters, the formulation containing CAE (FC) showed no significant increase in the sebum content under the same conditions (Figure $3)$.

After 28 days of application of formulation FC, there were no significant changes in biophysical and skin image analysis parameters: stratum corneum water content, microrelief and number of active sebaceous glands. A reduction of transepidermal water loss was observed, but it was not significant due to inter-individual variation.

The application of FC significantly reduced the number of pores on the chin of the volunteers after 28 days of use (Figure 3). humana utilizou-se o teste de normalidade Kolmogorov-Smirnov utilizando o software estatístico Origem 9.0. Para os efeitos após uma única aplicação e análise subjetiva, os dados foram analisados utilizando o teste T de Student para distribuição normal e o teste de Mann Whitney para distribuição não paramétrica. Para os efeitos a longo prazo, utilizou-se a análise paramétrica ANOVA com o pós-teste de Tukey e para a distribuição não-paramétrica, utilizou-se o teste de Kruskal-Wallis com Dunns. Os testes acima foram realizados utilizando GraphPad Prism 5.0. As diferenças foram aceites como estatisticamente significativas em $\mathrm{p}<0,05$.

\section{Resultados}

\section{Atividade antioxidante}

O CAE inibiu a quimioluminescência catalizada por HRP de uma maneira dose-dependente e mostrou actividade de remoção de radicais (Figura 1), com um valor de concentração de $8,63 \mathrm{mg} / \mathrm{mL}$ para $50 \%$ de inibição de radicais livres (IC50).

\section{Eficácia clínica}

Não foram obtidos efeitos imediatos significativos no conteúdo de água no estrato córneo, na perda transepidérmica de água e nos parâmetros do microrrelevo. A aplicação da formulação $\mathrm{F}$ (sem CAE) aumentou o nível de sebo após 2 e 4 horas de aplicação (Figura 2). No entanto, apesar de possuir os mesmos filtros UV, a formulação contendo CAE (FC) não mostrou aumento significativo no conteúdo de sebo nas mesmas condições (Figura 3).

Após 28 dias de aplicação da formulação $\mathrm{FC}$, não houve alterações significativas nos parâmetros de análise biofísica e de imagem da pele: conteúdo de água no estrato córneo, microrrelevo e número de glândulas sebáceas ativas. Observou-se redução na perda transepidérmica de água, mas não foi significativa devido à variação interindividual. A aplicação de $\mathrm{FC}$ reduziu significativamente o número de poros no queixo dos voluntários após 28 dias de uso (Figura 3). 


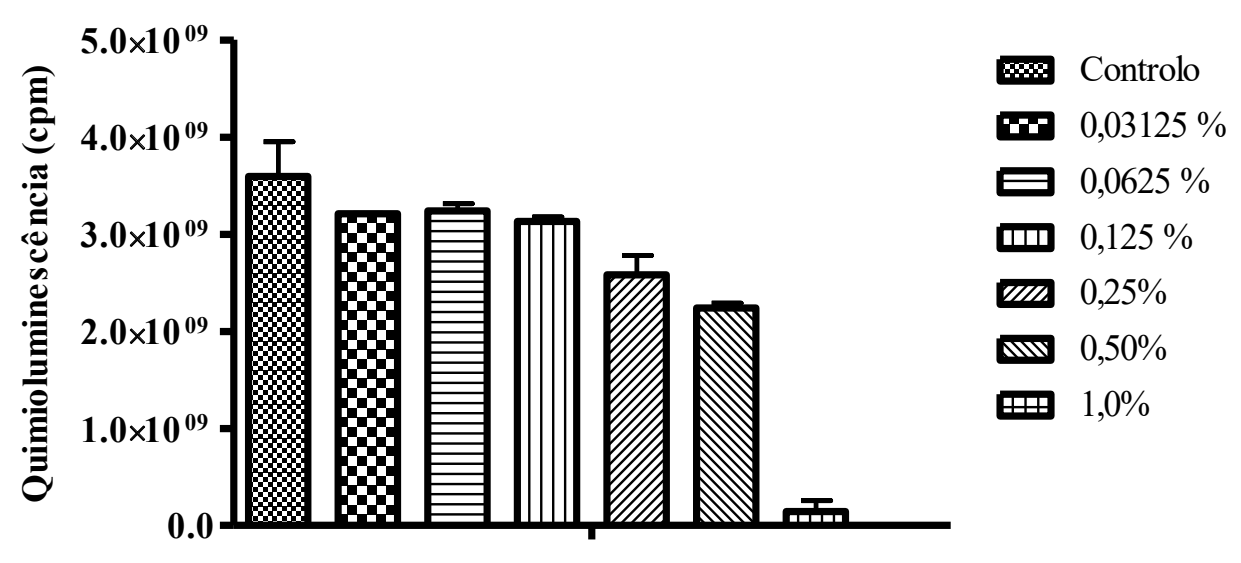

Concentrações

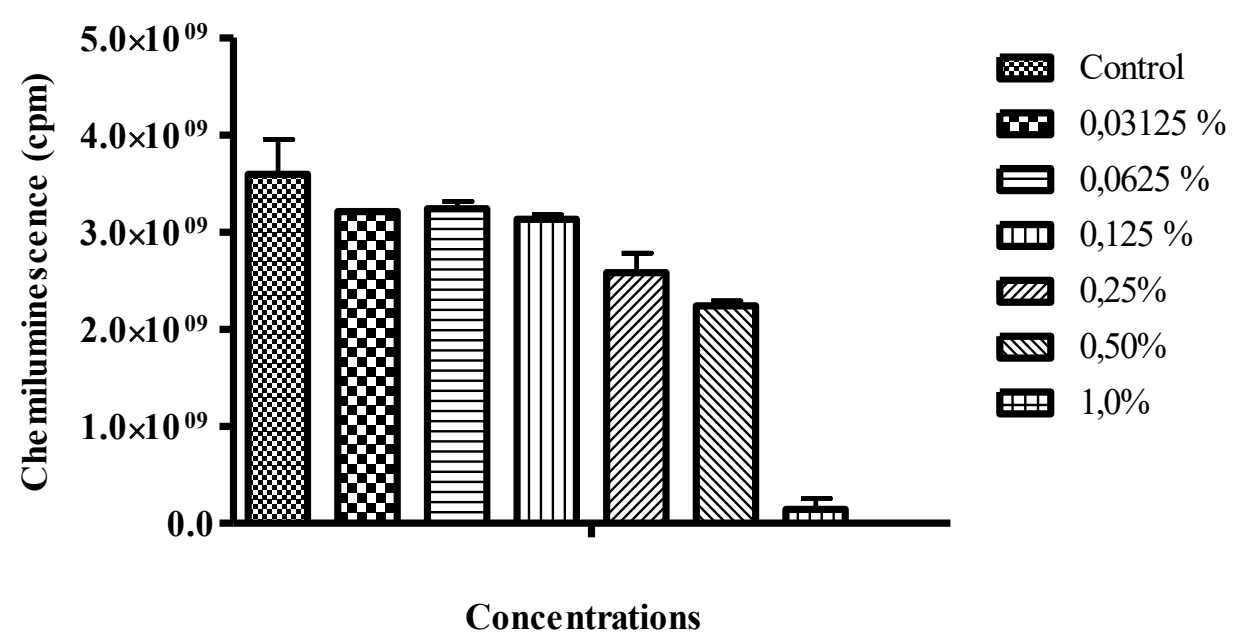

Figure 1/ Figura 1 - Inhibitory effect of the diluted solutions Cashew apple extract on reactive oxygen species generated by HRP-catalyzed luminescent reactions with luminol/ Efeito inibitório das soluções diluídas de extrato de maçã de caju (CAE) em espécies reativas de oxigénio geradas por reações luminescentes catalisadas por HRP com luminol 

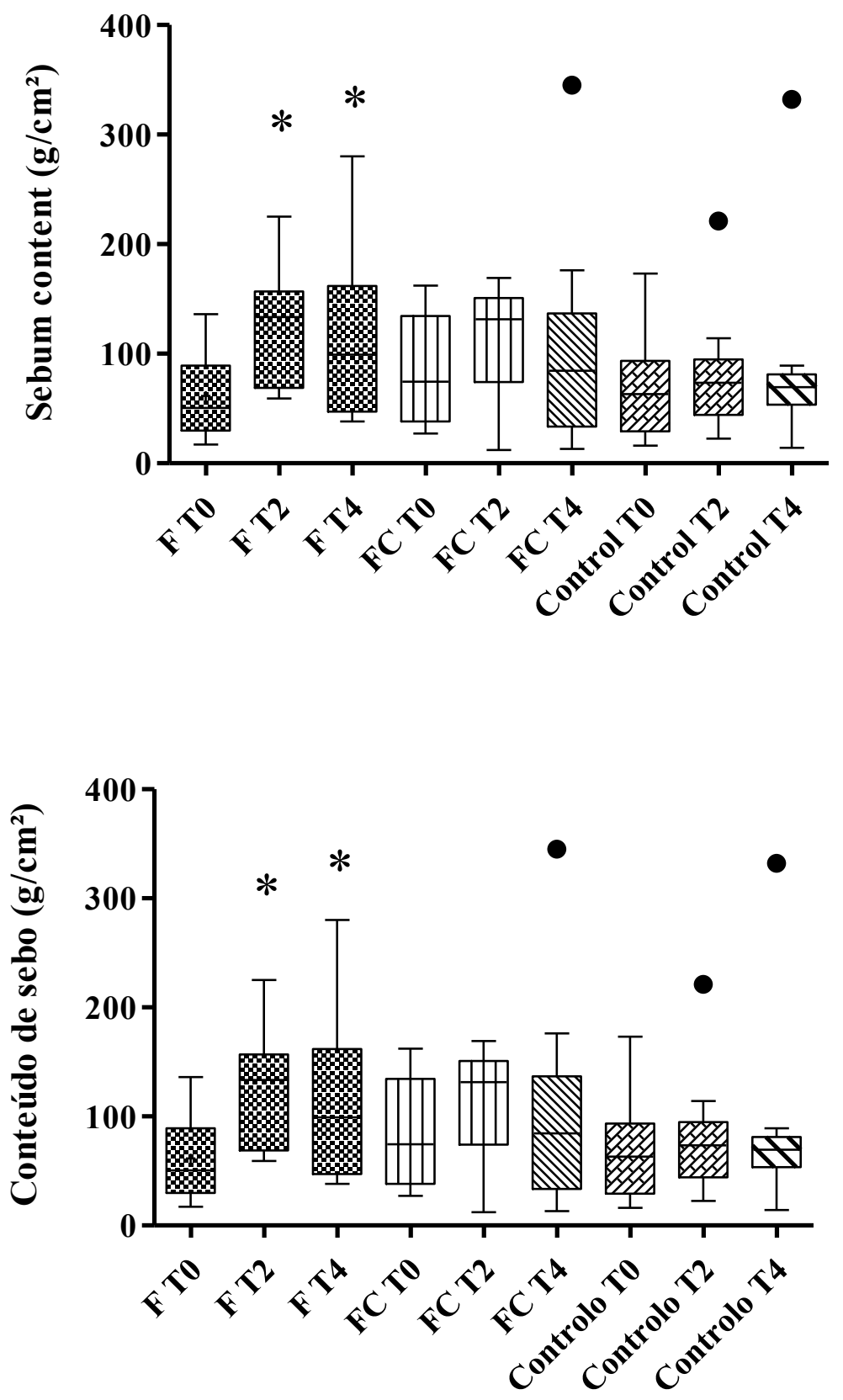

Figure 2/ Figura 2 - Sebum content, before (T0) and two (T2) and four hours (T4) after the application of the formulation $\mathrm{F}$ - sunscreen formulation and $\mathrm{FC}$ - sunscreen formulation $+3.5 \%$ cashew extract and in control region (Cont). (ANOVA test, $\mathrm{n}=12$ subjects, mean $\pm \mathrm{SD}$ ). * Significantly different from baseline values $(\mathrm{p}<0.05) /$ Teor de sebo antes de (T0), após duas (T2) e quatro horas (T4) da aplicação da formulação $\mathrm{F}$ - formulação de filtro solar e FC - formulação de filtro solar $+3.5 \%$ de extrato de caju e na região controlo. (Teste de ANOVA, $\mathrm{n}=12$ indivíduos, média $\pm \mathrm{DP}$ ). * Significativamente diferente dos valores basais $(\mathrm{p}<0,05)$. 
A)

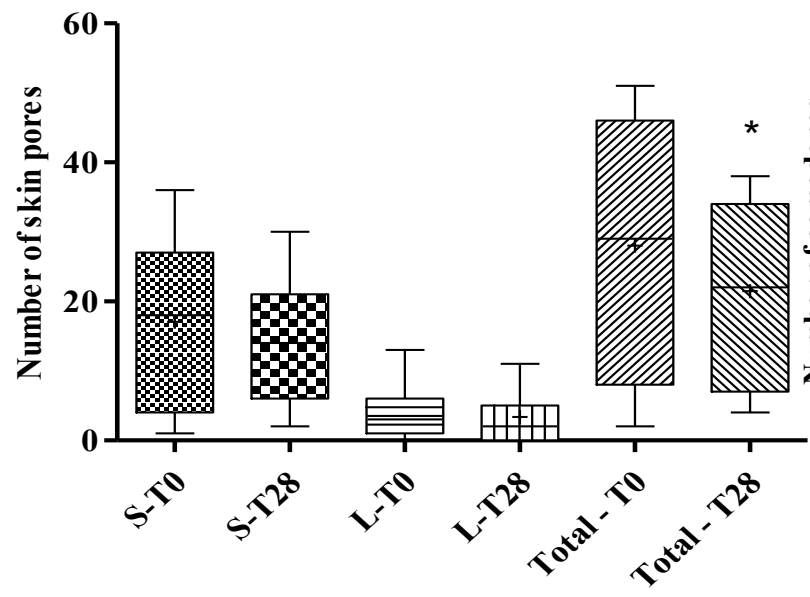

B)

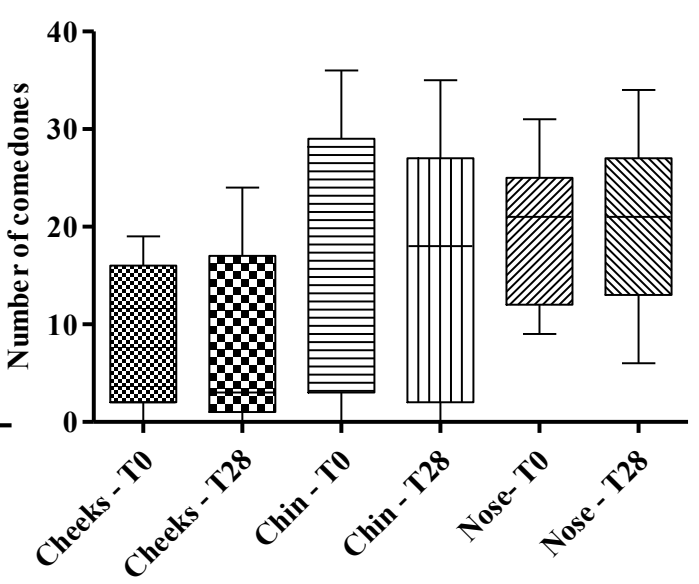

B)

A)
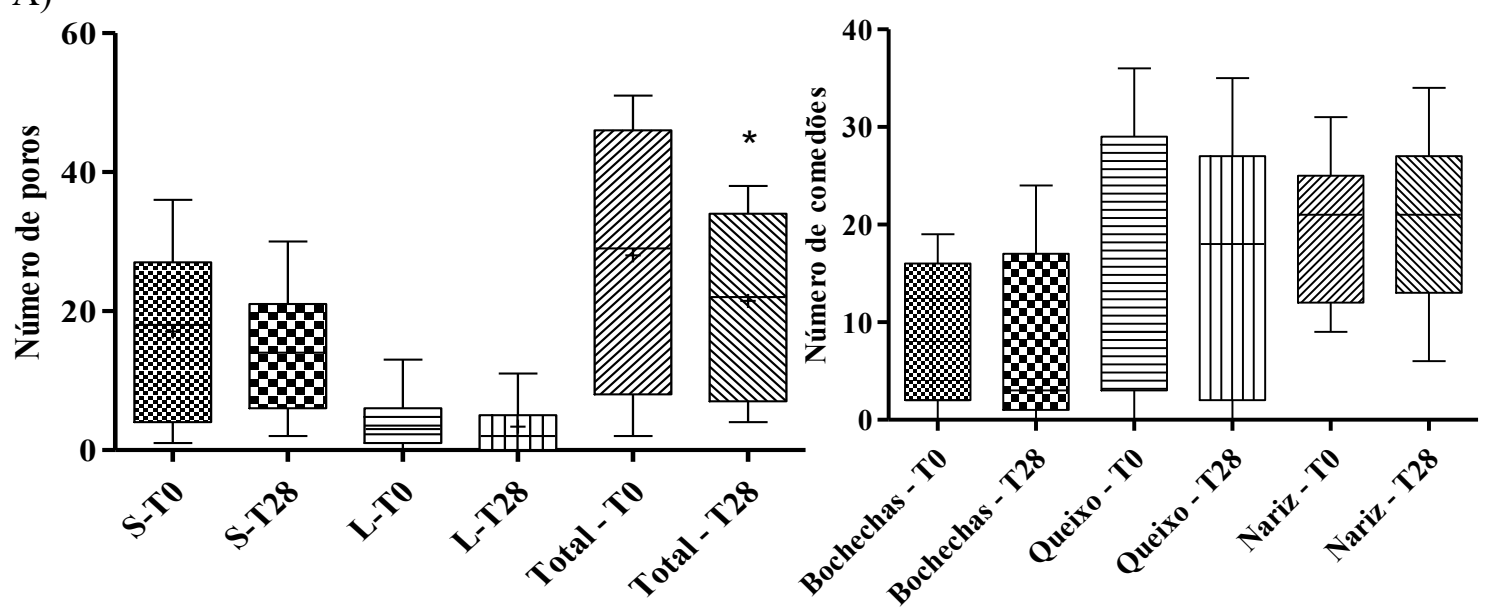

Figure 3/ Figura 3 - Number of small (S), large (L) and total pores on the chin (A) and number of comedones (B) before, and after 28 days of daily application of FC (FC - sunscreen formulation $+3.5 \%$ cashew extract).(Student $\mathrm{t}$ test, $\mathrm{n}=12$ subjects, mean $\pm \mathrm{SD})$. ${ }^{*}$ Significantly different from baseline values $(\mathrm{T} 0), \mathrm{p}<0.05)$./ Número de poros pequenos $(\mathrm{S})$, grandes $(\mathrm{L})$ e totais no queixo $(\mathrm{A})$ e número de comedões (B) antes, e após 28 dias de aplicação diária de FC (FC - formulação de filtro solar $+3,5 \%$ extrato de caju). (Teste $t$ de Student, $n=12$ indivíduos, média \pm DP). Significativamente diferente dos valores basais $(\mathrm{T} 0)(\mathrm{p}<0,05)$. 


\section{Sensory evaluation and purchase intention}

CAE further improved the sensory profile of the sunscreen formulation. FC attained good scores in sensory analysis in terms of sensation of touch and sensation of the touch after 5 minutes of application (Figure 4), when compared to the formulation F. FC received a score of $100 \%$ for purchase intention, while $\mathrm{F}$ received a score of $50 \%$.
Avaliação sensorial e intenção de compra

O CAE melhorou ainda mais o perfil sensorial da formulação de filtro solar. FC obteve boa pontuação na análise sensorial em termos de sensação de toque e sensação de toque após 5 minutos de aplicação (Figura 4), em comparação com a formulação F. FC recebeu $100 \%$ para intenção de compra, enquanto $\mathrm{F}$ recebeu $50 \%$.
A)

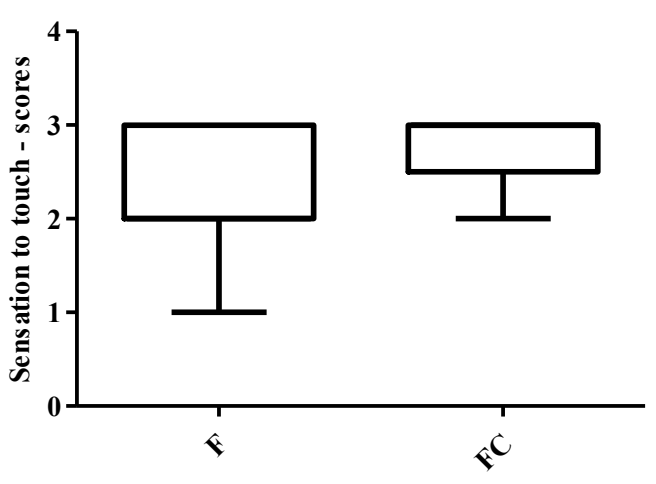

A)

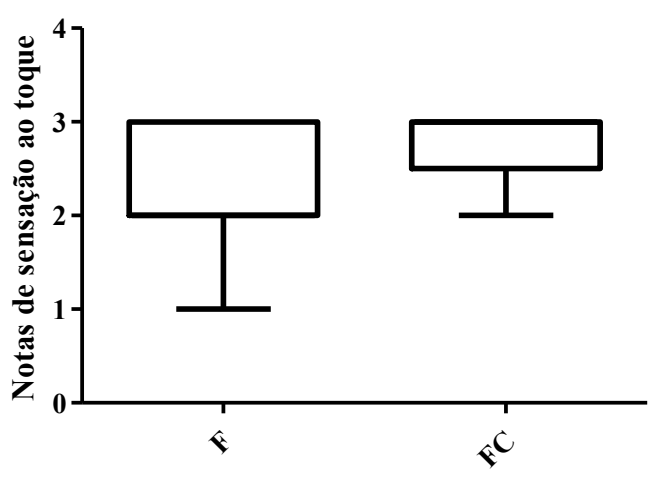

B)

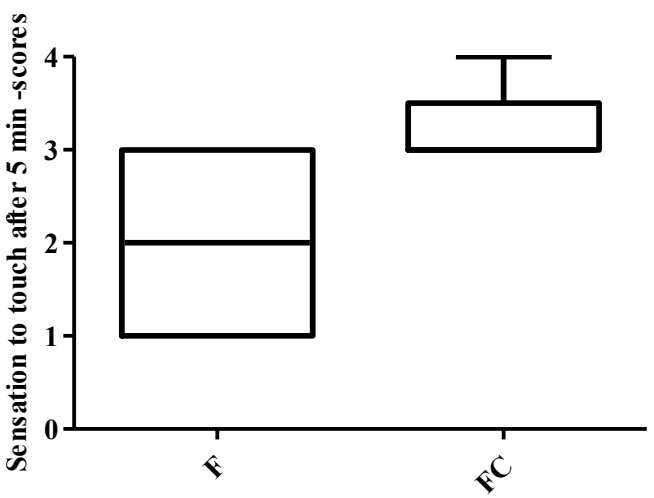

B)

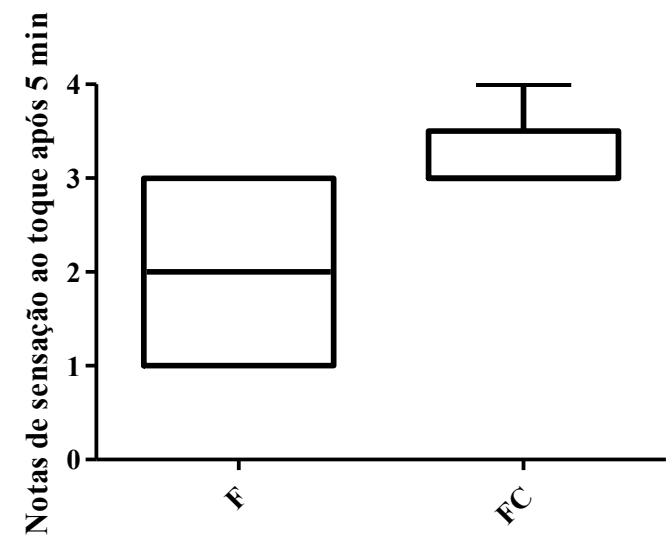

Figure 4/ Figura 4 - Box-plot representation of the scores obtained in the parameters: Sensation of touch (A) and Sensation of touch after 5 minutes (B) of the formulations F (sunscreen formulation) and FC (sunscreen formulation supplemented with 3.5\% of Cashew extract). The parameters were scored: (1) Bad, (2) Regular (3) Good, (4) Excellent. *Significantly different from F (Sunscreen Formulation)/ Gráfico de caixa das notas obtidas para sensação ao toque após 5 minutos (B) das formulações F (formulação de filtro solar) e FC (formulação de filtro solar suplementada com 3,5\% de extrato de caju). Os parâmetros foram pontuados: (1) Mau, (2) Regular (3) Bom, (4) Excelente. * Significativamente diferente de F (Formulação fotoprotetora). 


\section{Discussion}

Botanical extracts have been widely used in topical and cosmetic products and can provide moisturizing, antioxidant, anti-inflammatoryand emollient effects. Their characterization and study of their applications are essential for effective and safe use in topical formulations $^{(18)}$.

The findings obtained in the present in vitro assay demonstrate that the CAE presents antioxidant activity.

This effect can be related to many antioxidant substances that have been described in different Cashew apple extracts. Carotenoids from cashew apple by-product represent an interesting source of xanthophylls ${ }^{(19)}$, antioxidant monomeric phenolics, e.g., quercetin 3-O-galactoside, myricetin, quercetin ${ }^{(20)}$ and anthocyanins ${ }^{(21)}$.. In previous studies, cashew extract and juice presented pronounced antioxidant activity related to presence of polyphenolic compounds, tannic acid and Vitamin $\mathrm{C}^{(11,22)}$.

The antioxidant activity of substances in topical formulations is of great interests due to the use of these substances for the prevention of oxidative stress and, thus, their action in maintaining skin health and prevent skin ageing ${ }^{(23)}$. In oily skin, free radicals are related to inflammatory processes involved in the formation of acne lesions. Thus, the antioxidant activity of CAE can prevent and reduce acne lesions ${ }^{(24) .}$

In addition to antioxidant activity, CAE improved the sensory profile of the developed sunscreen formulation. Sensory characteristics of topical formulations greatly influence consumer's acceptance especially for oily skin types. Thus, the physical properties of the formulation could lead to a greater or lesser approval of the product $^{(17)}$.

Considering the lipophilic nature of the filters used to obtain the photoprotective effects, the development of a sunscreen with high SPF and differentiated sensory profile is a considerable challenge in cosmetic technology field. Thus, the sunscreen containing CAE is an innovative product, and CAE stands out as an active ingredient that can improve the sensory profile of the cosmetic formulations, mainly to oily skin.

Due to the astringent and constricting effects of the tannins present in $\mathrm{CAE}$, it can contribute to the retention of sebum in the infundibulum of the skin pores. This fact, can explain the findings of no increment of sebum content after a single application of FC.

After the 28-day-period of application, FC did not increase the number of comedones and therefore, it can be considered suitable for oily skin. The use of sunscreens has beenrelated to the development of cosmetic

\section{Discussão}

Os extratos botânicos têm sido amplamente utilizados em produtos tópicos e podem proporcionar efeitos hidratantes, antioxidantes, anti-inflamatóriose emolientes. A caracterização e estudo de suas aplicações são essenciais para o uso eficaz e seguro em formulações tópicas ${ }^{(18)}$.

Os resultados obtidos no presente ensaio in vitro demonstram que o CAE apresenta actividade antioxidante.

Este efeito pode estar relacionado a muitas substâncias antioxidantes que tem sido descritas em diferentes extratos de Caju. Os carotenoides do subproduto da polpa do caju representaram uma fonte interessante de xantofilas ${ }^{(19)}$, fenólicos monoméricos antioxidantes: 3-O-galactósido da quercetina, miricetina e quercetina ${ }^{(20)}, \mathrm{e}$ antocianinas $^{(21)}$ são alguns exemplos.Em estudo prévio, o extrato e suco de caju apresentaram actividade antioxidante pronunciada, relacionada com a presença de compostos polifenólicos, ácido tânico e vitamina $C^{(11,22)}$. A actividade antioxidante das substâncias em formulações tópicas é de grande interesse devido ao uso destas substâncias que podem prevenir o stress oxidativo e, assim, actuar na manutenção da saúde da pele e prevenir o envelhecimento da pele ${ }^{(23)}$. Na pele oleosa, os radicais livres estão relacionados com processos inflamatórios envolvidos na formação de lesões de acne. Esta atividade antioxidante CAE pode prevenir e reduzir as lesões de $\operatorname{acne}^{(24)}$.

Além da actividade antioxidante, CAE melhorou o perfil sensorial da formulação de protetor solar desenvolvida. As características sensoriais das formulações tópicas influenciam muito a aceitação do consumidor, especialmente para os tipos de pele oleosa. Desta forma, as propriedades físicas da formulação poderiam levar a uma maior ou menor aprovação do produto ${ }^{(17)}$. Considerando a natureza lipofílica dos filtros utilizados para obtenção da fotoproteção, o desenvolvimento de um filtro solar com FPS elevado e perfil sensorial diferenciado é um grande desafio no campo da tecnologia cosmética. Assim, o protetor solar contendo CAE é um produto inovador que se destaca como um ingrediente ativo que pode melhorar o perfil sensorial das formulações cosméticas, principalmente para a pele oleosa. Devido aos efeitos adstringentes e constritivos pelos taninos presentes no CAE, eles podem contribuir para a retenção de sebo no infundíbulo dos poros da pele. Este fato, pode explicar a razão de nenhum incremento de conteúdo de sebo após uma única aplicação de FC.

Após o período de 28 dias de aplicação, FC não aumentou o número de comedões e, portanto, pode ser 
acne, especially on oily skin. Thus, this finding is of great interest and importance for both dermatologists and consumers ${ }^{(25)}$.

Furthermore, FC significantly reduced the number of pores on the chin of the volunteers after 28 days of application. This can be attributed to the pore constriction of the tannins in this extract.

Aside from monomeric phenolics, tannins are the major phenolics of the cashew apple(22). The cashew apple tanins contains high percentages of (-)-epigallocatechin and (-)-epigallocatechin- O-gallate, followed by minor quantities of (-)-epicatechin and (-)-epicatechin-3-Ogallate phenols(26). The tannins, as mentioned, may be responsible for the improvement of sensory properties, as well as, for the pore constriction and sebum regulation, which are important effects for oily skin formulations. Thus, our results highlights the benefits of using CAE in cosmetic formulations to improve the appearance of pores, a very common feature in oily $\operatorname{skin}(5,6)$. CAE presented antioxidant activity, further improved the sensory properties of the sunscreen formulation and showed clinical effects in terms of oil control, pore constriction and improvement of skin conditions. Thus, the developed formulation could prompt the balance for the hydrolipidic film on skin and favor the maintenance of good skin physiological function, and improve the appearance of oily skin. Finally, this extract is presented as an innovative active ingredient to be applied in the development of topical products for skin protection with antioxidant activity and efficacy to improve oily skin conditions.

In summary, CAE further improved the sensory properties of the formulation, showed antioxidant activity and clinical efficacy in the control of oiliness and improvement of oily skin conditions. The potential of CAE use in topical formulations, primarily for oily skin was highlighted. Therefore, identification of beneficial health applications for the cashew can promote its use and have a significant socioeconomic impact on regions where it is produced.

\section{Acknowledgments}

The authors gratefully acknowledge the financial support of FAPESP (Fundação de Amparo a Pesquisa do Estado de Sao Paulo) and CAPES (Coordenação de Aperfeiçoamento de Pessoal de Nível Superior) for scholarship. considerado adequado para pele oleosa. O uso de protetores solares está relacionado ao desenvolvimento de acne cosmética, especialmente na pele oleosa. Assim, este achado é de grande interesse e importância tanto para dermatologistas como para consumidores ${ }^{(25)}$.

Além disso, FC reduziu significativamente o número de poros no queixo dos voluntários após 28 dias de aplicação, o que pode ser atribuído à constrição de poros pelos taninos deste extrato.

Além dos fenólicos monoméricos, os taninos são os principais fenólicos da maçã do caju ${ }^{(22)}$. Os taninos da polpa contêm altas porcentagens de (-) - epigalhocatequina e O-galhato de (-) - epigalhocatequina, seguidas de pequenas quantidades de fenóis de 3-O-galhato de $(-)$ - epicatequina ${ }^{(26)}$. Os taninos, como mencionado, podem ser responsáveis pela melhoria das propriedades sensoriais, bem como, para a constrição do poro e regulação do sebo, que são efeitos importantes para formulações de pele oleosa. Assim, nossos resultados apontam para os benefícios do uso de CAE em formulações cosméticas para melhorar a aparência dos poros, característica muito comum na pele oleosa ${ }^{(5,6)}$.

CAE apresentou actividade antioxidante, melhorou ainda mais as propriedades sensoriais da formulação de filtro solar e mostrou efeitos clínicos em termos de controlo de oleosidade, constrição de poros e melhoria das condições da pele. Desta forma, a formulação desenvolvida pode estimular o equilíbrio do filme hidrolipídico na pele e favorecer a manutenção de uma boa função fisiológica da pele e melhorar a aparência da pele oleosa. Finalmente, este extrato é apresentado como um ingrediente ativo inovador para ser aplicado no desenvolvimento de produtos tópicos para proteção da pele com actividade antioxidante e eficácia para melhorar as condições da pele oleosa.

Em resumo, o CAE melhorou ainda mais as propriedades sensoriais da formulação, mostrou actividade antioxidante e eficácia clínica no controlo da oleosidade e melhoria das condições oleosas da pele. O potencial de uso de CAE em formulações tópicas, principalmente para pele oleosa foi destacado. Portanto, a identificação das aplicações benéficas à saúde do caju pode promover seu uso e ter um impacto socioeconómico significativo.

\section{Agradecimentos}

Os autores agradecem o apoio financeiro da FAPESP (Fundação de Amparo a Pesquisa do Estado de São Paulo) e da CAPES (Coordenação de Aperfeiçoamento de Pessoal de Nível Superior). 


\section{Conflict of interest}

The authors declare that there is no financial or personal relationship that can be understood as representing a potential conflict of interest.

\section{Conflito de interesses}

Os autores declaram que não há relação financeira ou pessoal que possa ser entendida como representando um potencial conflito de interesses.

\section{References/ Referências}

[1]. Oliveira VH. Cashew crop. Rev Bras Frutic 2008; 30:01-03.

[2]. Rodrigues F, Gaspar C, Palmeira-de-Oliveira A, Sarmento B, Amaral MH, Oliveira BPP. Application of coffee silverskin in cosmetic formulations: physical/antioxidant stability studies and cytotoxicity effects. Drug Devel Ind Pharm 2016; 42:99-106.

[3]. Yahaya LE, Aliyu OM, Hammed LA, Aroyeun SO. Variation in the physicochemical characteristics of cashew (Anacardium occidentale L.) apples with maturity. Br Food J 2010; 112:93-99.

[4]. Agostini-Costa TS, Lima A, Lima, MV. Determinação de tanino em pedúnculo de caju: método da vanilina versus método do butanol ácido. Quím Nova 2003; 6:763-765.

[5]. Bele AA, Jadhav VM, Kadam VJ. Potential of tannins: a Review. Asian J Plant Sci 2010; 9:209-214.

[6]. Smith M. 2006. Toners and Astringents. In: Cosmetic Formulation of Skin Care Products (1st ed). Informa Healthcare.

[7]. Silva, PK, Farias GA, Araújo ER, Sapucay, MJLC, Colares, PNQ, Pedroza CM, Rêgo, ER, Rêgo MM. Caracterização física e química de genótipos de caju. Horticultura Brasileira 2009; 27:1551-1555.

[8]. Garruti DS, Franco MRB, Silva MAAP, Janzantti NS, Alves GL. Evaluation of volatile flavour compounds from cashew apple (Anacardium occidentale L) juice by gas chromatography/olfactometry technique. J Sci Food Agric 2003; 83:1455-1462.

[9]. Talasila U, Shaik KB. Quality, spoilage and preservation of cashew apple juice: A review. Int J Food Sci Tech 2013; 52:54-62.

[10]. Kubo I, Nihei K, Tsujimoto K. Antibacterial action of anacardic acids against Methicillin Resistant Staphylococcus aureus (MRSA). J Agric Food Chem 2003; 51: 7624-7628.

[11]. Kubo I, Masuoka N, Ha TJ, Tsujimoto K. Antioxidant activity of anacardic acids. Food Chem 2006; 99:555-562.

[12]. Kubo I, Ha TJ, Tsujimoto K, Tocoli FE, Green I R. Evaluation of lipoxygenase inhibitory activity of anacardic acids. Z Naturforsch C 2008; 63:539-546.

[13]. Maia Campos PMBG, Gianeti MD, Kanashiro A, Lucisano-Valim YM, Gaspar LR. In vitro antioxidant and in vivo photoprotective effects of an association of bioflavonoids with liposoluble vitamins. Photochem Photobiol 2006; 82:683-688.

[14]. Nóbrega AT, Wagemaker TAL, Maia Campos PMBG. Antioxidant activity of Matricaria chamomilla L. extract and clinical efficacy of cosmetic formulations containing this extract and its isolated compounds. Biomed Biopharmac Res 2012; 10:249-261.

[15]. Maia Campos PMBG, Mercurio DG. 2013. Emulsão Fotoprotetora. Patent: Innovation Privilege. Register number: BR 10 2013 008117-5, Date: 04.04.13, Institution of Register: Instituto Nacional da Propriedade Industrial - I.N.P.I./S.P.

[16]. Mercurio DG, Segura JH, Demets MB, Maia Campos PMBG. Clinical scoring and instrumental analysis to evaluate skin types. Clin Exp Dermatol $2013 ; 38: 302-308$.

[17]. Marcon AFVS, Wagemaker TAL, Maia Campos PMBG. Rheology, clinical efficacy and sensorial of a silicone-based formulation containing pearl extract. Biomed Biopharm Res 2014; 11:247-255.

[18]. Fisk WA, Lev-Tov HA, Sivamani RK. Botanical and phytochemical therapy of acne: a systematic review. Phytother Res 2014; $28: 1137-52$.

[19]. Abreu FP, Dornier M, Dionisio AP, Carail M, Caris-Veyrat C, Dhuique-Mayer C. Cashew apple (Anacardium occidentale L.) extract from by-product of juice processing: A focus on carotenoids. Food Chem 2013; 138:25-31.

[20]. Michodjehoun-Mestres L, Souquet J-M, Fulcrand H, Bouchut C, Reynes M, Brillouet J-M. Monomeric phenols of cashew apple (Anacardium occidentale L.). Food Chem 2009; 112:851-857.

[21]. da Silva LMR, de Figueiredo EAT, Silva RNM, Pinto Vieira, IG, Wilane de Figueiredo R, Brasil IM, Gomes CL. Quantification of bioactive compounds in pulps and by-products of tropical fruits from Brazil. Food Chem 2014; 14:398-404

[22]. Cavalcante AAM, Rubensam G, Picada JN, Silva EG, Moreira AJCF, Henriques JC. Mutagenicity, antioxidant potential, and antimutagenic activity against hydrogen peroxide of cashew (Anacardium occidentale) apple juice and cajuina. Environ Mol Mutagen 2003; 41:360-369.

[23]. Masaki H. Role of antioxidants in the skin: anti-aging effects. J Dermatol Sci 2010;58:85-90.

[24]. Dreher F, Maibach H. 2001. Protective effects of topical antioxidants in humans. Curr Probl Dermatol 2001; 29:157-64.

[25]. Guerrero D. Dermocosmetic approach to acne by the dermatologist. Annales de dermatologie 2010; 137:29-32.

[26]. Michodjehoun-Mestre L, Souquet JM, Fulcrand H, Bouchu C, Reynes M, Brillouet JM. Monomeric phenols of cashew apple (Anacardium occidentale L.). Food Chem 2009; 112:851-857. 